\title{
A novel approach to assess pulmonary function in patients with chronic obstructive pulmonary disease using tissue velocity imaging
}

\author{
Xiao-Zhi Zheng1, Jing Wu1, Xu-Yan Tan² \\ ${ }^{1}$ Department of Ultrasound, The First People's Hospital of Yancheng, Yancheng , ${ }^{2}$ Department of Ultrasound, Jiangsu \\ Province Tumor Hospital, Nanjing, Jiangsu Province, P.R. China
}

\begin{abstract}
Aims: To explore the feasibility of quantitative evaluation of pulmonary function in patients with chronic obstructive pulmonary disease (COPD) using tissue velocity imaging (TVI) and strain rate imaging (SRI) via transthoracic lung ultrasonography. Material and methods: Eighty inpatients with clinically diagnosed COPD underwent pulmonary function test and transthoracic lung ultrasound on the same day. Lung ultrasound variables and pulmonary function parameters were analyzed. Results: All patients with COPD had faster breathing and significant reduced lung function compared with healthy participants $(\mathrm{p}<0.05)$. The lung ultrasound parameters, velocity ( $\max -\mathrm{min}, \mathrm{cm} / \mathrm{s})$, displacement (max-min, $\mathrm{mm})$, strain (max-min, $\%)$ and strain rate (max-min, 1/s) were significantly higher in patients with COPD $(\mathrm{p}<0.05)$. A good negative correlation was found between lung ultrasound variables and pulmonary function parameters in patients with COPD. Stepwise multiple regression analysis indicated that the velocity (max-min, $\mathrm{cm} / \mathrm{s}$ ) was the only independent determinant of FEV1/FVC (\%). With the use of $\mathrm{FEV} 1 / \mathrm{FVC}<70 \%$ as the criteria of irreversible pulmonary function impairment to distinguish an abnormal pulmonary function, the area under the ROC was 0.99 for the velocity (max-min, $\mathrm{cm} / \mathrm{s}$ ) of the lung tissue in the process of breathing. The sensitivity, specificity, positive predictive value, negative predictive value and accuracy of the cut off value $(1.19 \mathrm{~cm} / \mathrm{s})$ was $97.63 \%, 100 \%, 100 \%, 80 \%$, and $98 \%$, respectively $(\mathrm{p}<0.001)$. Conclusions: Tissue velocity imaging via transthoracic lung ultrasound is a useful modality in the assessment of pulmonary function in patients with COPD.
\end{abstract}

Keywords: lung ultrasound, tissue velocity imaging, strain rate imaging, pulmonary function test, chronic obstructive pulmonary disease

\section{Introduction}

Pulmonary function testing plays an important role in the assessment of disease severity, functional disability, disease activity, and response to treatment, particularly with regard to diseases such as chronic obstructive pulmonary disease (COPD), asthma, and interstitial

Received 24.10.2015 Accepted 01.12.2015

Med Ultrason

2016, Vol. 18, No 2, 177-181

Corresponding author: Xu-Yan Tan, Department of Ultrasound,

Jiangsu Province Tumor Hospital,

42 Baiziting Road, Nanjing 210009

Jiangsu Province, P.R. China

Phone: $+86025-83283364$

Fax: $+86025-83283364$

E-mail: tanxuyanzxz@163.com lung disease [1]. However, pulmonary function testing requires cooperation between the subjects and the examiner, and the results obtained depend on technical as well as personal factors [2]. Alternative and noninvasive techniques which do not require the patient's cooperation during spontaneous breathing are required.

In the past two decades, tissue velocity imaging (TVI) and strain rate imaging (SRI) have been used in the quantitative evaluation of myocardial function and this has been a recommended component of routine echocardiography $[3,4]$. However, whether TVI and SRI can quantify pulmonary function remains unknown. The objective of this study was to evaluate the value of TVI and SRI in the assessment of pulmonary function and to compare them with the results of pulmonary function testing in patients with COPD. 


\section{Material and methods}

\section{Study population}

Eighty inpatients with clinically diagnosed COPD (42 men, 38 women; $60.6 \pm 17.2$ years, range: 43-79 years), and 80 age and sex-matched healthy volunteers (40 men, 40 women; $59.8 \pm 16.5$ years, range: $42-80$ years) were investigated between February 2011 and December 2014 in the Department of Respiratory Medicine of our institution. The COPD criteria is based on a spirometry test (forced expiratory volumes in 1 second [FEV1]/forced vital capacity $[\mathrm{FVC}]<70 \%$ ) according to the Global Initiative for Chronic Obstructive Lung Disease $[5,6]$. The study was approved by the local Ethics Committee of Yancheng (No.2014052), and free informed consent was obtained from all the subjects. The pulmonary function test and transthoracic lung ultrasound were performed on the same day.

\section{Pulmonary function test}

Standard spirometry were performed in all patients by means of a fully equipped computerized system (Cosmed SrL, Quark PFTs ergo, P/N Co9035 -12-99, Italy) for measurement of FEV1, FVC, FEV1/FVC, total lung capacity (TLC), tidal volume (VT), maximal voluntary ventilation (MVV), expiratory reserve volume (ERV), vital capacity (VC), peak expiratory flow (PEF), forced expired flow at $25 \%$ of FVC (FEF $25 \%$ ), and volume backextrapol \% FVC.

\section{Transthoracic lung ultrasound}

All the patients underwent transthoracic examination using a commercially available Vivid E9 ultrasound machine (GE Healthcare, Horten, Norway) equipped with a M5S single-crystal matrix-array transducer and Q-analyze quantitative analysis software for the analysis of TVI and SRI. The scanning was performed by two experienced operators with 20 years experience in lung ultrasonography and echocardiography who were blinded to all clinical data and previous reading. The intercostal spaces were used as acoustic windows. Patients were placed in a sitting position raising their arms above the head to widen the intercostal space and facilitate scanning. First of all, the TVI and SRI function were activated, and the dynamic images of three respiratory cycles in four chest zones (the upper anterior zone, the lower anterior zone, the upper lateral zone, and the basal lateral zone) per side were stored at a frame rate of 50 frames per second for subsequent analysis. Then, a region of interest was placed at each zone. The maximal velocity, minimal velocity, maximal displacement, minimal displacement, maximal strain, minimal strain, maximal strain rate and minimal strain rate during the process of breathing were measured (fig 1). The difference between maximal and minimal velocity $(\mathrm{V}$, max-min, $\mathrm{cm} / \mathrm{s}$ ), the difference between maximal and minimal displacement $(\mathrm{D}$, max-min, $\mathrm{mm}$ ), the difference between maximal and minimal strain (St, max-min, \%) and the difference between maximal and minimal strain rate (SR, max-min, 1/s) were calculated. Each variable per zone was obtained by averaging measurements from three successive respiratory cycles, and each variable in all zones was also obtained by averaging measurements from all zones.

\section{Echocardiography}

A complete bidimensional and Doppler study was performed in all subjects using the ultrasound scanner mentioned above. Patients were placed in the left lateral decubitus position and standard parasternal and fourchamber apical views were obtained by two independent operators (above mentioned). The pulmonary artery systolic pressure (PASP) was estimated by adding the right atrial pressure to the transtricuspid pressure gradient in the absence of right ventricular outflow obstruction $[7,8]$. The transtricuspid pressure gradient was calculated from the peak tricuspid regurgitation velocity using the modified Bernoulli equation. Measurement of cardiac morphology and function indicators included: left atrial end-systolic anteroposterior diameter (LA), left ventricular end-diastolic anteroposterior diameter (LV), right atrial end-systolic transverse diameter (RA), right ventricular end-diastolic anteroposterior diameter (RV), tricuspid annular plane systolic excursion (TAPSE), left

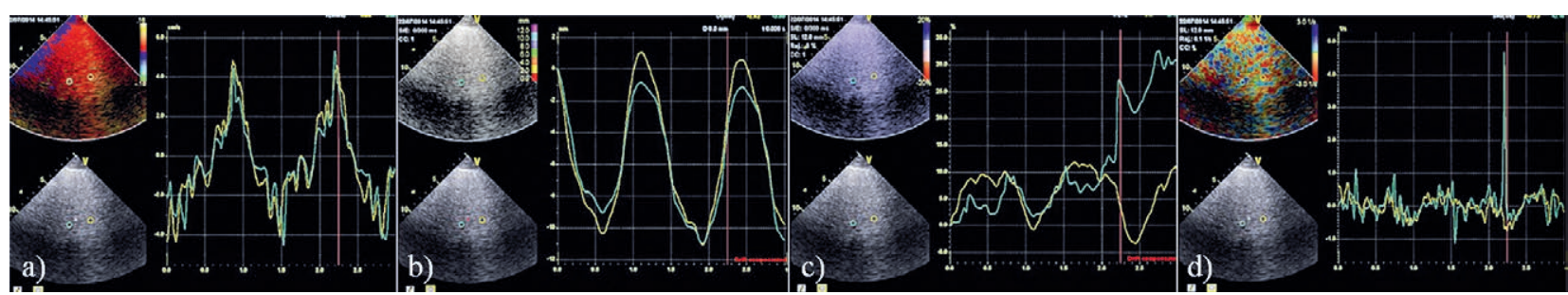

Fig 1. Measurement of lung velocity, displacement, strain and strain rate: a) the maximal velocity and minimal velocity determined by tissue velocity imaging; b) the maximal displacement and minimal displacement determined by using tissue velocity imaging; c) the maximal strain and minimal strain determined by strain imaging; d) the maximal strain rate and minimal strain rate determined by strain rate imaging. 
ventricular ejection fraction (LVEF). All values for each parameter were obtained by averaging measurements from three successive cardiac cycles.

\section{Statistical analysis}

Results in this study are expressed as mean \pm standard deviation or number and percentage. The differences between the two groups were tested using an unpaired two tailed $t$ test. The correlation between different parameters was performed using the Pearson test. A receiver operating characteristic curve (ROC) analysis was used to predict irreversible pulmonary function impairment and to determine the optimal cut-off points and validity parameters. A value of $\mathrm{p}<0.05$ was considered statistically significant. All statistical analyses were performed with SPSS version 16 software for Windows (SPSS Inc, Chicago, IL).

\section{Results}

\section{Baseline characteristics}

As shown in Table I, all patients with COPD had faster breathing and heartbeat, shortened six minutes walking distance, larger right atrial and ventricular size, increased pulmonary artery systolic pressure, significant reduced lung function and TAPSE compared with healthy participants $(\mathrm{p}<0.01$ or $\mathrm{p}<0.05)$, while the lung ultrasound variables, V (max-min, $\mathrm{cm} / \mathrm{s}$ ), D (max-min, $\mathrm{mm}$ ), St (max-min, \%), and SR (max-min, 1/s) were all significantly higher in patients with COPD comparing with healthy participants. No significant differences were found between the two groups with regard to age, male ratio, left atrial and ventricular size, as well as LVEF $(\mathrm{p}>0.05)$.

\section{Comparison of the lung ultrasound variables among different chest zones}

As shown in Table II, there were no significant differences among different chest zones with regard to the lung ultrasound variables, V (max-min, $\mathrm{cm} / \mathrm{s}), \mathrm{D}$ (max-min, $\mathrm{mm})$, St (max-min, \%) and SR (max-min, 1/s) $(\mathrm{p}>0.05)$.
Table I. Clinical, echocardiographic, lung ultrasound and pulmonary function parameters of healthy participants and patients with chronic obstructive pulmonary disease (COPD).

\begin{tabular}{lll}
\hline Variables & $\begin{array}{l}\text { Healthy } \\
\text { subjects }\end{array}$ & $\begin{array}{l}\text { Patients with } \\
\text { COPD }\end{array}$ \\
\hline Clinical parameters & & \\
Age, years & $66.8 \pm 10.9$ & $67.8 \pm 12.5$ \\
Males, n (\%) & $28(52)$ & $30(54)$ \\
BF, times/min & $15.6 \pm 3.7$ & $26.5 \pm 7.9 * *$ \\
HR, beats/min & $71.59 \pm 10.66$ & $86.19 \pm 20.19^{*}$ \\
Six minutes walking distance, m & $531(492-588)$ & $259(136-359)^{* *}$ \\
Echocardiographic parameters & & \\
RV, mm & $19.08 \pm 3.64$ & $26.92 \pm 6.42^{*}$ \\
RA, mm & $31.29 \pm 6.89$ & $43.56 \pm 14.01^{*}$ \\
LV, mm & $28.36 \pm 13.29$ & $31.49 \pm 12.58$ \\
LA, mm & $31.25 \pm 8.81$ & $32.37 \pm 10.69$ \\
PASP, mmHg & $16.28 \pm 7.56$ & $58.77 \pm 19.69^{* *}$ \\
TAPSE, mm & $20.88 \pm 5.67$ & $12.89 \pm 4.69^{*}$ \\
LVEF, \% & $70.33 \pm 16.28$ & $65.28 \pm 17.55$ \\
Lung ultrasound parameters & & \\
V, max-min, cm/s & $0.59 \pm 0.17$ & $2.58 \pm 1.69^{* *}$ \\
D, max-min, mm & $0.49 \pm 0.19$ & $2.71 \pm 1.53^{* *}$ \\
St, max-min, \% & $1.78 \pm 0.67$ & $5.91 \pm 2.34^{* *}$ \\
SR, max-min, 1/s & $0.46 \pm 0.19$ & $0.85 \pm 0.36^{*}$ \\
Lung function parameters & & \\
VT, \% & $83.79 \pm 13.63$ & $67.88 \pm 14.05^{*}$ \\
MVV, \% & $87.88 \pm 14.95$ & $60.23 \pm 16.89^{*}$ \\
ERV, \% & $90.23 \pm 16.36$ & $73.68 \pm 19.25^{*}$ \\
FVC, \% & $78.2 \pm 20.69$ & $66.97 \pm 13.59^{*}$ \\
FEV1, \% & $92.33+32.57$ & $50.98 \pm 16.34^{*}$ \\
FEV1/FVC, \% & $117.19 \pm 25.68$ & $75.89 \pm 10.66^{*}$ \\
FEF25, \% & $85.79 \pm 16.55$ & $77.63 \pm 19.15^{*}$ \\
PEF, \% & $89.12 \pm 17.98$ & $69.17 \pm 18.44^{*}$ \\
Volume backextrapol \% FVC & $86.53 \pm 18.34$ & $71.59 \pm 19.67^{*}$ \\
TLC, \% & $98.95 \pm 16.27$ & $71.78 \pm 12.23^{*}$ \\
\hline
\end{tabular}

$* * \mathrm{p}<0.01, * \mathrm{p}<0.05$. LA, left atrial end-systolic anteroposterior diameter; LV, left ventricular end-diastolic anteroposterior diameter; $\mathrm{RA}$, right atrial end-systolic transverse diameter; RV, right ventricular end-diastolic anteroposterior diameter; PASP, pulmonary artery systolic pressure; TAPSE, tricuspid annular plane systolic excursion; LVEF, left ventricular ejection fraction; BF, breathing frequency; V, velocity; D, displacement; St, strain; SR, strain rate; max, maximal; min, minimal; VT, tidal volume; MVV, maximal voluntary ventilation; ERV, expiratory reserve volume; FEV1, forced expiratory volumes in 1 second; FVC, forced vital capacity; VC, vital capacity; PEF, peak expiratory flow; FEF $25 \%$, forced expired flow at $25 \%$ of FVC; TLC, total lung capacity.

Table II. Comparison of the lung ultrasound parameters among different chest zones in patients with chronic obstructive pulmonary disease.

\begin{tabular}{|c|c|c|c|c|}
\hline Variables & Upper anterior zone & Lower anterior zone & Upper lateral zone & Basal lateral zone \\
\hline \multicolumn{5}{|l|}{ Left side } \\
\hline $\mathrm{V}, \max -\min , \mathrm{cm} / \mathrm{s}$ & $2.66 \pm 1.77$ & $2.55 \pm 1.58$ & $2.68 \pm 1.66$ & $2.49 \pm 1.70$ \\
\hline $\mathrm{D}, \mathrm{max}-\mathrm{min}, \mathrm{mm}$ & $2.80 \pm 1.24$ & $2.85 \pm 1.39$ & $2.79 \pm 1.62$ & $2.88 \pm 1.55$ \\
\hline $\mathrm{St}, \max -\min , \%$ & $5.93 \pm 2.55$ & $5.91 \pm 2.46$ & $5.86 \pm 2.36$ & $5.88 \pm 2.29$ \\
\hline $\mathrm{SR}, \max -\min , 1 / \mathrm{s}$ & $0.87 \pm 0.51$ & $0.84 \pm 0.39$ & $0.86 \pm 0.42$ & $0.84 \pm 0.36$ \\
\hline \multicolumn{5}{|l|}{ Right side } \\
\hline $\mathrm{V}, \max -\mathrm{min}, \mathrm{cm} / \mathrm{s}$ & $2.57 \pm 1.66$ & $2.61 \pm 1.76$ & $2.59 \pm 1.47$ & $2.57 \pm 1.59$ \\
\hline $\mathrm{D}, \mathrm{max}-\mathrm{min}, \mathrm{mm}$ & $2.69 \pm 1.48$ & $2.81 \pm 1.49$ & $2.73 \pm 1.43$ & $2.72 \pm 1.44$ \\
\hline St, $\max -\min , \%$ & $5.94 \pm 2.29$ & $5.83 \pm 2.35$ & $5.92 \pm 2.46$ & $5.95 \pm 2.61$ \\
\hline $\mathrm{SR}, \max -\min , 1 / \mathrm{s}$ & $0.89 \pm 0.56$ & $0.85 \pm 0.54$ & $0.87 \pm 0.41$ & $0.85 \pm 0.43$ \\
\hline
\end{tabular}

V, velocity; D, displacement; St, strain; SR, strain rate; max, maximal; min, minimal 
Table III. Linear correlation analysis for pulmonary function and lung ultrasound variables in patients with chronic obstructive pulmonary disease.

\begin{tabular}{lllll}
\hline Variables & $\begin{array}{l}\text { V, max-min, cm/s } \\
\text { r (p-value) }\end{array}$ & $\begin{array}{l}\text { D, max-min, mm } \\
\text { r(p-value) }\end{array}$ & $\begin{array}{l}\text { St, max-min, \% } \\
\text { r(p-value) }\end{array}$ & $\begin{array}{l}\text { SR, max-min, 1/s } \\
\text { r (p-value) }\end{array}$ \\
\hline VT, \% & $-0.945(0.000)$ & $-0.901(0.006)$ & $-0.930(0.002)$ & No sig. \\
MVV, \% & $-0.998(0.000)$ & $-0.939(0.002)$ & $0.975(0.000)$ & No sig. \\
ERV, \% & $-0.969(0.000)$ & $-0.921(0.003)$ & $-0.952(0.001)$ & No sig. \\
FEV1/FVC, \% & $-0.995(0.000)$ & $-0.998(0.002)$ & $-0.973(0.000)$ & $-0.843(0.017)$ \\
TLC, \% & $-0.930(0.002)$ & No sig. & $-0.917(0.004)$ & No sig. \\
FEF25, \% & $-0.920(0.003)$ & No sig. & No sig. & No sig. \\
PEF, \% & $-0.905(0.005)$ & No sig. & $-0.908(0.005)$ & No sig. \\
Volume backextrapol \%FVC & $-0.938(0.002)$ & No sig. & $0.905(0.005)$ & $-0.952(0.001)$ \\
\hline
\end{tabular}

No sig., no statistical significance; VT, tidal volume; MVV, maximal voluntary ventilation; ERV, expiratory reserve volume; FEV1, forced expiratory volumes in 1 second; FVC, forced vital capacity; PEF, peak expiratory flow; FEF $25 \%$, forced expired flow at $25 \%$ of FVC; V, velocity; D, displacement; St, strain; SR, strain rate; max, maximal; min, minimal

Table IV. Multiple regression analysis (Method: stepwise) for forced expiratory volumes in 1 second/forced vital capacity (FEV1/ FVC) and related lung ultrasound variables in patients with chronic obstructive pulmonary disease.

\begin{tabular}{|c|c|c|c|c|c|c|}
\hline Independent variable & $\mathbf{R}$ & $\mathbf{F}$ & p value & $\beta$ coefficient & t value & p value \\
\hline $\mathrm{V}, \max -\min$ & 0.999 & 19.74 & $<0.0001$ & -0.999 & -42.123 & $<0.0001$ \\
\hline
\end{tabular}

$\beta$ coefficient, standardized coefficient; $V$, velocity; max, maximal; min, minimal

\section{Correlation between lung ultrasound variables and pulmonary function parameters in patients with COPD}

As shown in Table III, a good negative correlation could be found between lung ultrasound variables and pulmonary function parameters in patients with COPD. The V (max-min, cm/s), D (max-min, mm), St (max-min, $\%$ ), and SR (max-min, 1/s) significantly correlated with FEV1/FVC $(\%)(p<0.001)$, while the V (max-min, $\mathrm{cm} / \mathrm{s})$ significantly correlated with all the pulmonary function parameters: VT(\%), MVV(\%), ERV(\%), FEV1/FVC(\%), TLC $(\%)$, FEF25(\%), PEF(\%), volume backextrapol $\% \mathrm{FVC}$. However, no lung ultrasound variables were associated with pulmonary function parameters.

\section{Multiple regression analysis}

As shown in Table IV the multiple regression analysis (stepwise method) indicated that $\mathrm{V}$ (max-min, $\mathrm{cm} / \mathrm{s}$ ) was an independent determinant of FEV1/FVC (\%).

\section{ROC analysis}

At FEV1/FVC $<70 \%$ (as criteria of irreversible pulmonary function impairment) the area under the ROC was 0.99 (95\% confidence interval: 0.98 to 1.01$)$ for $\mathrm{V}$ (max-min, $\mathrm{cm} / \mathrm{s}$ ) of the lung tissue in the process of breathing. The sensitivity, specificity, positive predictive value, negative predictive value, and accuracy of the cut off value $(1.19 \mathrm{~cm} / \mathrm{s})$ was $97.63 \%, 100 \%, 100 \%, 80 \%$, and $98 \%$, respectively $(\mathrm{p}<0.001)$.

\section{Inter/intra observer variability}

Intra observer and inter observer agreement in the measurement of lung ultrasound variables were fairly good: intraclass correlation coefficient (ICC) was 0.92
(0.71-0.97), $0.93(0.75-0.98)$ for inter and intra observer, respectively. Inter and intra observer agreement in the measurement of pulmonary function parameters, were still good: ICC was 0.93 (0.67-0.98), 0.93 (0.71-0.99) for inter and intra observer, respectively.

\section{Discussions}

The results of our study indicate that transthoracic lung ultrasound can effectively detect the pulmonary function changes, and the increased difference between maximal and minimal velocity obtained at tissue velocity imaging is a sensitive and specific predictor of pulmonary function impairment in patients with COPD.

Recently, the COPD diagnostic criteria based on spirometry test (FEV1/ FVC $<70 \%$ ) has been questioned, and many factors, such as age, race, gender, or BMI were related with FEV1/ FVC [9-11]. In addition, pulmonary function testing requires good laboratory quality assurance, highly motivated and well-trained physicians and technologists. Therefore, it is necessary to explore simple, new, and better ways of improving patient outcomes.

TVI and SRI maturation, which have been developed to quantify myocardial velocity, deformation and deformation rate, respectively, just open an interesting window into the lung tissue movement mechanics. However, the lung is one of the major body organs, and there is no unified standard for the choice of the acoustic window. In our study, we borrowed from the methods described by Gargani et al [12] and Volpicelli et al [13] namely, eight chest zones, four chest zones (the upper anterior zone, the lower ante- 
rior zone, the upper lateral zone and the basal lateral zone) per side. Surprisingly, we found there were no significant differences among different chest zones with regard to the lung ultrasound variables in patients with COPD.

COPD is characterized by irreversible chronic airflow limitation that is caused by emphysematous destruction of lung elastic tissue and/or obstruction in the small airways due to occlusion of their lumen by inflammatory mucus exudates, narrowing and obliteration [14]. In our study, we found that all patients with COPD had faster breathing, increased difference between maximal and minimal velocity, displacement, stain, and stain rate. We think these findings resulted from the compensatory effects of the respiratory muscle, and TVI and SRI actually reflected a passive movement of lung tissue. We also found a good negative correlation between lung ultrasound variables and pulmonary function parameters in patients with COPD, the worst pulmonary function, the greater difference between maximal and minimal velocity, displacement, stain, and stain rate. Through the statistical analysis, we confirmed that the difference between maximal and minimal velocity was the only independent determinant of FEV1/FVC, with a good diagnosis performance for pulmonary function impairment (FEV1/ $\mathrm{FVC}<70 \%$ ) at a cut off value of $1.19 \mathrm{~cm} / \mathrm{s}$.

This study had some limitations. Firstly, lung ultrasound is an operator-dependent method and there is a lack of unified criteria to perform this examination more precisely. Secondly, the lung tissue movement characteristics expressed with TVI and SRI need to be further interpreted. Thirdly, the patient population examined was limited. New data need to be collected in subsequent studies.

\section{Conclusions}

This is the first study on evaluating the feasibility of transthoracic lung ultrasound in the assessment of pulmonary function in patients with COPD. Our study demonstrates that the increased difference between maximal velocity and minimal velocity obtained tissue velocity imaging is a sensitive and specific predictor of pulmonary function impairment in patients with COPD. Although there are some limitations mentioned above, this method still holds considerable clinical promise for the assessment of pulmonary function.

Acknowledgements: The authors gratefully acknowledge the technical assistance and helpful sugges- tions from Li-Zhu Miao, You Zhou, Xiao Wang, Wen-Yan Zhou, Xiao-Qin Huang at the Ultrasound Department, The First People's Hospital of Yancheng, Jiangsu Province, P.R.China.

\section{Conflict of interest: none}

\section{References}

1. Ruppel GL, Enright PL. Pulmonary function testing. Respir Care 2012; 57: 165-175.

2. Tojo N. Problems of lung function testing in the laboratory. Rinsho Byori 2006; 54: 834- 837.

3. Thijssen JM, de Korte CL. Cardiological Ultrasound Imaging. Curr Pharm Des 2014; 20: 6150-6161.

4. Pauliks L.Tissue doppler myocardial velocity imaging in infants and children--a window into developmental changes of myocardial mechanics. Echocardiography 2013; 30: $439-446$.

5. Pistelli R, Ferrara L, Misuraca C, Bustacchini S. Practical management problems of stable chronic obstructive pulmonary disease in the elderly. Curr Opin Pulm Med 2011; 17 Suppl 1: S43-S48.

6. Obase Y, Mouri K, Shimizu H, et al. Nutritional deficits in elderly smokers with respiratory symptoms that do not fulfill the criteria for COPD. Int J Chron Obstruct Pulmon Dis 2011; 6: 679-683.

7. McQuillan BM, Picard MH, Leavitt M, Weyman AE. Clinical correlates and reference intervals for pulmonary artery systolic pressure among echocardiographically normal subjects. Circulation 2001; 104: 2797-2802.

8. Finkelhor RS, Lewis SA, Pillai D. Limitations and strengths of doppler/echo pulmonary artery systolic pressure-right heart catheterization correlations:a systematic literature review. Echocardiography 2015; 32: 10-18.

9. Melbye H, Medbø A, Crockett A. The FEV1/FEV6 ratio is a good substitute for the FEV1/FVC ratio in the elderly. Prim Care Respir J 2006; 15: 294-298.

10. Quanjer PH, Brazzale DJ, Boros PW, Pretto JJ. Implications of adopting the Global Lungs Initiative 2012 all-age reference equations for spirometry. Eur Respir J 2013; 42: 1046-1054.

11. O'Donnell DE, Deesomchok A, Lam YM, et al. Effects of BMI on static lung volumes in patients with airway obstruction. Chest 2011; 140: 461-468.

12. Gargani L, Volpicelli G. How I do it: lung ultrasound. Cardiovasc Ultrasound 2014; 12: 25.

13. Volpicelli G, Mussa A, Garofalo G, et al. Bedside lung ultrasound in the assessment of alveolarinterstitial syndrome. Am J Emerg Med 2006; 24: 689-696.

14. Ojo O, Lagan AL, Rajendran V, et al. Pathological changes in the COPD lung mesenchyme-Novel lessons learned from in vitro and in vivo studies. Pulm Pharmacol Ther 2014; 29: 121-128. 